\title{
The dental camera for your practice or lab
}

Creating professional photo documentations after only a short learning curve? No problem - with Shofu EyeSpecial, the dedicated dental camera. The EyeSpecial has everything your dental practice or laboratory really needs. It possesses smart special features, is easy to operate, reproducibly takes excellent images, and its use can be delegated to your colleagues without any lengthy training.

The Shofu EyeSpecial has built-in photographic expertise. The camera relieves users of their worries about ring flash, aperture, depth of field etc and has everything needed to easily take informative patient images - without any specialist knowledge of photography or additional equipment. Thanks to its smart integrated features, the EyeSpecial reliably produces excellent photos, without any time-consuming alignment of flashes or other settings and adjustments.

Like no other camera, the EyeSpecial supports modern hygiene practices in your operatory. The completely smooth camera body can be quickly and thoroughly disinfected between two patients, and menu navigation on the touch panel works properly even when wearing disposable gloves.
This ultralight high-performance camera does not require any heavy accessories. It can easily be held with one hand, freeing the other to hold a cheek retractor or a mirror. And the integrated flash system provides the optimal light, irrespective of the ambient light conditions.

Just like its predecessor, the new EyeSpecial C-IV has special shooting modes for ease of use and quicker results. Thanks to the autofocus feature, you can concentrate on subject and shutter or, even better, delegate photo documentations to your colleagues. The camera now offers a video feature for case documentations in 'motion pictures' and an optional cross polariser, allowing you to look inside a tooth without any light reflections.

Would you like to learn more about the new EyeSpecial C-IV? The product brochure can be downloaded at https://www.shofu.de/ en/produkt/eyespecial-c4-uk/. If you would like to see a demonstration at your practice or laboratory and take advantage of an attractive offer, your Shofu representative will be happy to assist you. Just contact the Shofu UK office on 01732783580 or sales@shofu.co.uk,www. shofu.co.uk.

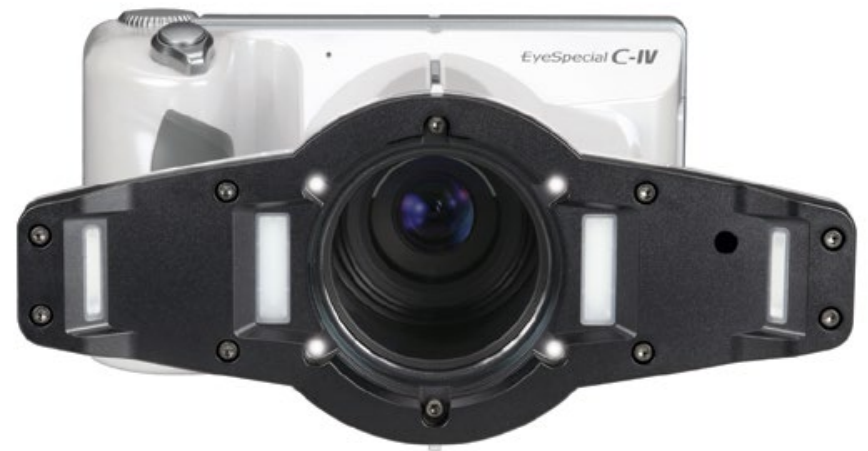

\section{Save time and money on sharpening instruments}

Finally every dental practice can easily determine how much money is wasted on sharpening instruments. The required time per week and the hourly wage are entered on the website www.am-eagle.org/ sharpening_calculator and the online tool then evaluates the expenses incurred each year.

In a second step, the financial benefits of switching to sharpen-free American

Eagle XP instruments can be discovered. These scalers and curettes retain the shape and functionality throughout their lifetime and are always ready with sharp cutting edges. The therapist can therefore noticeably reduce the physical effort and act more ergonomically. Thanks to XP Technology, sharpening stones are history and time can now be used more productively.

The effort to resharpen the equipment is often underestimated, but the procedure requires considerable experience, patience and the proper technique. The instruments must be aligned and fixed, and a gauge is needed to maintain the correct angle. To

\section{POC COVID-19 test for dentistry}

Dental Life Sciences/Introdent have been appointed the exclusive distributor, for dentistry, of the AbC-19 TM Rapid Test developed by UK Rapid Test Consortium (UK-RTC), part of the UK government's five pillar national testing strategy for COVID-19.

The AbC-19 TM Rapid Test antibody test has achieved sensitivity of $100 \%$ (95\% confidence interval $92.6 \%$ to $100 \%$ ) and specificity of $98.4 \%$ (95\% confidence interval $95.4 \%$ to $99.7 \%$ ). AbC-19TM Rapid Test has obtained CE certification. Production lines are being geared up to produce commercial quantities in early September. The test will indicate the presence of antibodies in 20 minutes. It is a simple easily administered point of care (POC) test. Regular and widespread testing is needed to combat COVID-19.

Depending on government requirements, Dental Life Sciences/ Introdent will be able to supply dentists and the dental supply industry as production capacity comes on stream. Dental Life Sciences will be introducing four further tests including a saliva-based test for antigens, later this year.

For more information, visit https:// youtu.be/WqT2z8jCEHs. To receive priority supplies and updates, register on the Introdent website, https://www. introdent.com/.

avoid facets and ensure a perfect working edge every detail must be checked with a magnifying glass.

Moreover, resharpened instruments along with substance also lose their ideal shape which is no longer comparable with the original design. This shortens the lifespan of the instruments, because it degrades their performance or they even become entirely useless and must be replaced. For more information on XP Technology from American Eagle visit www.am-eagle.de/en/xp-technology. 